East African Medical Journal Vol. 77 No. 3 March 2000

CROSS-CULTURAL ATTITUDES TOWARDS SUICIDE AMONG SOUTH AFRICAN SECONDARY SCHOOL PUPILS

K. Peltzer, Ph.D., Department of Psychology, V. I. Cherian, Ph.D. and L. Cherian, Ph.D, Department of Psychology of Education, University of the North, Private Bag X1106, Sovenga 0727, South Africa

Request for reprints to: Prof. Karl Peltzer, Department of Psychology, University of the North, Private Bag X1106, Sovenga 0727, South Africa

\title{
CROSS-CULTURAL ATTITUDES TOWARDS SUICIDE AMONG SOUTH AFRICAN SECONDARY SCHOOL PUPILS
}

\author{
K. PELTZER, V. I. CHERIAN and L. CHERIAN
}

\begin{abstract}
Objective: To investigate the attitudes towards suicide among Grade II secondary school pupils among three cultural groups in South Africa.

Design: Cross sectional study.

Setting: Grade II Secondary school pupils chosen at random from three urban schools in Pietersburg.

Participants: The sample included 366 pupils, 150 (41\%) males and $216(59 \%)$ females, the mean age was 19.3 years ( $\mathrm{SD}=2.6$ ), with a range from 17 to 24 years. The three cultural groups were 142 blacks, 112 whites and 112 Asians.

Main outcome measures: Socioeconomic and family background (14 items), suicide data (4 items), and a 30-item Multi-Attitude Suicide Tendency Scale for Adolescents (MAST-12). Results: Suicide ideation and plans to commit suicide are the highest among Asians, closely followed by Whites and lowest among Black pupils. The frequency of attempted suicide was lower among Blacks (11.3\%) than that among Asians (13.5\%) and Whites (13\%). Analysis of variance indicated a significantly higher score among suicide attempters on attraction to death and repulsion by life and a significantly lower score on attraction to life and repulsion by death. Furthermore, this study found a significant correlation between total MAST, suicide ideation, suicide intent, history of completed suicide in family or friend, parents divorced, family size and suicide attempt.

Conclusion: This study found differential effects across diverse ethnocultural adolescent groups for suicidal ideation, plants and attempts. This gives indications on how suicide prevention programmes can be constructed in culture-congenial ways.
\end{abstract}

\section{INTRODUCTION}

Suicide is one of the leading causes of death for young people in industrial and developing countries(1-3). Suicide attempts rather than completed suicides have increased most in the youngest age group(4). The Department of Education(5) in the Northern Province of South Africa reports high rates of suicide and para-suicide among secondary school pupils. A history of previous suicidal behaviour in the form of one or more non-fatal suicide attempts has emerged as the most powerful predictor of suicide $(2,4)$. It is therefore self-evident that an increasing number of studies have been assembling knowledge on non-fatal suicidal behaviour in adolescents. The majority of these studies, however, have used psychiatric based samples and their results cannot therefore be generalized to normal secondary school pupils. In addition, the sociocultural context across different ethnocultural groups has not been studied. Sociocultural context factors on parasuicide in Africa have been identified as socioeconomic status, age, gender and ethnicity(3,6-8). Spirito et al (9) noted sex differences among adolescent suicide attempters.
In South Africa, cultural groups have played a central theme in their historical experience(10). This study was, therefore, conducted to evaluate the prevalence of suicidal ideation, plans or attempts among the different ethnocultural groups as such data are hardly available.

\section{MATERIALS AND METHODS}

Sample: The study population comprised Grade II secondary school pupils chosen at random from three urban schools in Pietersburg. The pupils (366) were 150 (41\%) male, and 216 (59\%) female, in the age range of 17 to $24 \mathrm{yrs}$ (mean age $19.3 \mathrm{yrs}$, $\mathrm{SD}=2.6$ ). The three cultural groups were 142 Blacks, 112 Whites and 112 Asians.

Instruments: The questionnaire included 10 items on socioeconomic background: age, sex, religion, ethnicity, occupation, education and income of parents; and four items on family background: parental status, family size, birth order. Moreover, the survey questionnaire included four items: one on suicidal ideation (lifetime), one on suicide intent (lifetime), one on suicide attempt (lifetime), and one on the history of completed suicide of a family member or friend(11). Finally, a 30-item Multi-Attitude Suicide Tendency Scale for Adolescents (MAST- 
A)(12), nine items on Repulsion by Death, seven items on Attraction to Life, seven items on Repulsion by Life, and seven items on Attraction to Death, was included. Statements are such as, "Many problems can be solved by death only," "I feel happy", "Death frightens me", and "None really loves me", each of which represents one of the four attitudes towards life and death. Participants indicate the degree to which they agree or disagree using a Likert scale from one (strongly disagree) to 5 (strongly agree). The scores ranged from 30 to 150 , and Cronbach alpha and split half reliability coefficient for the MAST-A was .7 for this sample. Factor structure indicates that $62.6 \%$ of the variance can be explained by the four factors already indicated above. Osman et al (13) found with the MAST-A a factor structure that explains $53.8 \%$ of the variance by four factors.

\section{RESULTS}

Table 1 indicates the frequency of lifetime suicidal behaviour by pupils, and their friends or relatives by culture. Suicide ideation and plans to commit suicide were highest among Asians, closely followed by Whites and lowest among Black pupils. The frequency of attempted suicide was lower among Blacks $(11.3 \%)$ than that among Asians (13.5\%) and Whites (13\%). A history of a family member or friend who died by suicide was also less frequent among Blacks (17.6\%) than that among Asians (19.8\%) and Whites (19.4\%).

Table 1

Lifetime suicidal behaviour by pupils and their friends or relatives by culture

\begin{tabular}{lccr}
\hline Item description & Black & White & Asian \\
$\mathrm{n}=142(\%)$ & $\mathrm{n}=112(\%)$ & $\mathrm{n}=112(\%)$
\end{tabular}

1. Have you thought about suicide or killing yourself? $\quad 10(7) \quad 47(43.5) \quad 49(44.1)$

2. Were there times when you intended to commit suicide? 18 (12.7) $26(24.1) \quad 27(24.3)$

3. Have you actually tried to commit suicide? $16(11.3) \quad 14(13) \quad 15(13.5)$

4. Did a family member or friend die by suicide?

$25(17.6) \quad 21(19.4) \quad 22(19.8)$

Table 2 indicates the mean comparisons across the MAST subscales by suicide attempters and non-suicide attempters.

Table 2

Mean comparisons across the MAST subscales by suicide attempters and non-suicide attempters

\begin{tabular}{llllr}
\hline Item description & $\begin{array}{l}\text { Suicide } \\
\text { attempters } \\
(\mathrm{n}=46) \\
\text { Mean (SD) }\end{array}$ & $\begin{array}{l}\text { Non-suicide } \\
\text { attempters } \\
(\mathrm{n}=320) \\
\text { Mean (SD) }\end{array}$ & $\mathrm{F}$ & P \\
\hline Repulsion by death & $2.96(.59)$ & $3.21(.46)$ & 3.73 & .05 \\
Attraction to life & $2.12(.65)$ & $2.59(.78)$ & 9.94 & .001 \\
Repulsion by life & $2.74(.79)$ & $2.32(6.7)$ & 7.63 & .006 \\
Attraction to death & $3.02(.90)$ & $2.42(.64)$ & 15.6 & .0001 \\
\hline
\end{tabular}

Table 3

Correlations between independent variables and suicide attempters

\begin{tabular}{llr}
\hline Independent variable & $\mathrm{r}$ & $\mathrm{P}$ \\
\hline Total MAST & .35 & $<.0001$ \\
Suicide ideation (lifetime) & .37 & $<.0001$ \\
Suicide intent (lifetime) & .52 & $<.0001$ \\
History of completed suicide in family & & \\
or friend (lifetime) & .19 & $<.009$ \\
Parents divorced & .17 & $<.03$ \\
Family size & .24 & $<.05$ \\
Gender & -.01 & $<.87$ \\
Age & -.0015 & $<.97$ \\
Socioeconomic status & -.08 & $<.60$ \\
Position in the family & .006 & $<.92$ \\
Life status of parents & .02 & $<.70$ \\
\hline
\end{tabular}

Analysis of variance indicated significantly higher scores among suicide attempters on Attraction to Death and Repulsion by Life and a significantly lower score on Attraction to Life and Repulsion by Death. Table 3 indicates the correlations between independent variables and suicide attempters. It is shown that 'suicide attempters' had a significant correlation of (i) .35 with total MAST ( $\mathrm{p}<.0001)$; (ii) .37 with suicide ideation $(\mathrm{p}<.0001)$; (iii) .52 with suicide intent $(\mathrm{p}<.0001$ ); (iv) .19 with a history of completed suicide in family or friend ( $\mathrm{p}<.009)$; (v) .17 with parents divorced ( $\mathrm{p}<.03$ ) and; (vi) .24 with family size $(\mathrm{p}<.05)$. Age, gender, socioeconomic status, position in family, and life status of parents do not have a correlation with suicide attempters.

\section{DISCUSSION}

Parasuicide prevalence rates: A high incidence of lifetime parasuicide rate was reported among the various cultural groups (Asians 13.5\%, Whites 13\%, and Blacks $11.3 \%$ ) for this urban secondary school sample. However, the reported life-time parasuicide rate among Black pupils $(11.3 \%)$ was lower than that of $15 \%$ among rural secondary school pupils in South Africa(14) and lower than that of $14.3 \%$ as found among Ethiopian grade 9-11 adolescents in Addis Ababa high schools(3). Similar findings were also reported among American junior and high school students with $14 \%$ lifetime suicide attempt and $47 \%$ life time suicide ideation (the White pupils in this sample also had $47 \%)(15)$.

Platt(16) has indicated that there is a social transmission effect in parasuicide. More investigations are needed to explore the high incidence of parasuicide. The prevalences of suicidal ideations, plans, and attempts in this sample of secondary school pupils suggest that suicidal behaviours are a significant mental health problem and that this problem varies to some extent by ethnocultural background of the pupils(10). Previous studies have shown males to be at a higher risk for completed suicides females for attempted suicides(10). However, this study did not find any significant gender difference regarding parasuicide(4). 
Determinants of suicidal attempts: This study found a significant correlation between total MAST, suicide ideation, suicide intent, history of completed suicide in family or friend, parents divorced, family size and suicide attempt. Age, gender, socioeconomic status, position in family, and life status of parents did not have a correlation with suicide attempters.

A correlation of .37 between suicide ideation and suicide attempters means that more than $13 \%$ of the variance in suicide attempters could be explained by variation in suicide ideation. Similarly suicide intent, history of completed suicide in family, parents divorced and family size could explain $3.6 \%, 2.9 \%$ and $5.8 \%$ of the variance respectively in suicide attempters. Suicide intent has a moderate correlation of .57 with suicide attempters, suicide ideation has a definite but slight correlation of .37, with suicide attempters. Parents divorced has the almost negligible correlation of .17 with suicide attempters. Family size has a definite but slight correlation of .24 with suicide attempters.

Kebede and Ketsela(3) found no significant associations between suicide attempts and age, sex, family history of suicide, parental education level and heavy alcohol intake among urban Ethiopian high school students. Other similar studies found a relationship between knowing someone (a friend or family member) who had completed suicide and suicide attempters $(15,17)$. However, Roberts et al (10) found that among American adolescents (grades 6-8) also age and "being worse off economically" were associated with suicide attempt. In line with Gutierrez et al (18) this study found that exposure to attempted suicide resulted in higher scores on the MAST.

Sociocultural differences: There have almost been no community-based epidemiological studies of suicidal behaviours among adolescents comparing diverse ethnocultural groups(10). Our results suggest that being Asian or White increases the risk for suicidal behaviours in South Africa, but also that the effects vary depending on the outcome examined (ideation vs. attempts vs. plans) and the group studied. Comparing lifetime suicide attempts between the three South African cultural groups [Blacks (11.3\%), Whites (13.0\%), and Asians (13.5\%) (mainly Muslims)] with Anglo American (11.0\%), African American $(8.7 \%)$ and Pakistani American (7.8\%) adolescents(10), is found that the South African youths have higher rates of suicide attempt. The comparatively low level of suicide attitudes and suicide attempts among the African group as compared to Whites and Asians may be attributable to low levels of suicide acceptability within the African society(19). Gijana et al (20) found in a Black South African sample that $90 \%$ expressed a negative attitude toward taking one's own life.

This study also corroborates studies that report increased risk of suicidal behaviour among Asian and White youths in South Africa. For example, Wassenaar et al (21) found high rates of suicidal behaviour among South African Asian women, and it is argued that it can be understood in the context of sociocultural transition. Our results suggest that risks of suicidal behaviours vary somewhat, depending on the ethnic group and the indicator of suicidal behaviours. That is, there were differential effects across groups observed for suicidal ideation, plans and attempts, which concurs with Roberts et al (10). This will give indications of how suicide prevention programmes can be constructed in culture-congenial ways(22).

\section{REFERENCES}

1. Ackerman, G.L. A congressional view of youth suicide. Amer. Psychologist. 1993; 48:183-184.

2. Diekstra, R.F. The epidemiology of suicide and parasuicide. Acta Psychiatrica Scandinavia. 1993; 9:371.

3. Kebede, D., and Katsela, T. Suicide attempts in Ethiopian adolescents in Addis Ababa high schools. Ethiop. Med. J. 1993; 31:83-89.

4. Diekstra, R.F.W. and Gulbinat W. The epidemiology of suicidal behaviour: a review of three continents. Wrld. Hlth. Stat. Quart. 1993; 46:52-68.

5. Department of Education. Northern Province, South Africa: Report on priority problems among Secondary School students, Pietersburg: Department of Education, 1997.

6. Morano, C.D., Cisler, R.A., and Lemerond, J. Risk factors for adolescent suicidal behavior: loss, insufficient familial support and hopelessness. Adolescence. 1993; 28:851-854.

7. Naidoo, P., and Pillay, B.J. Parasuicide in a general hospital in South Africa. Psychological Reports. 1993; 72:979-981.

8. Ndosi, N.K., and Waziri, M.C. The nature of parasuicide in Dar es Salaam, Tanzania. Soc. Sci. Med. 1997; 44:55-61.

9. Spirito, A., Bond, A., Kurkjian, J., Devost, L., Bosworth, T., and Brown, L.K. Gender differences among adolescent suicide attempters. Crisis. 1993; 14:178-183.

10. Roberts, R.E., Chen, Y.R. and Roberts, C.R. Ethnocultural differences in prevalence of adolescent suicidal behaviours. Suicide and LifeThreatening Behaviour. 1997; 27:208-217.

11. Buddeberg, C., Buddeberg-Fischer, B., Gnam, G., Schmid, J., and Christen, S. Suicidal behavior in Swiss students: an 18 months followup survey. Crisis. 1996; 17:78-84.

12. Orbach, I., Milstein, I., Har-Even, D. Apter, A., Tiano, S., and Elizur, A. A Multi-attitude Suicide Tendency Scale for adolescents. Psychological Assessment. J. Clin. Psychol. 1991; 3:398-404.

13. Osman, A., Barrios, F.X., Panak, W.F., Osman, J.R., Foffman, J. and Hammer R. Validation of the Multi-Attitude Suicide Tendency Scale in adolescent samples. J. Clin. Psychol. 1994; 50:847-855.

14. Peltzer, K., Cherian, V.I. and Cherian, L. Attitudes toward suicide among South African secondary school pupils. Psychological Reports (In press).

15. Wagner, B.M., Cole, R.E. and Schwartzman, P. Psychosocial correlates of suicide attempts among junior and senior high school youth. Suicide and Life-Threatening Behaviour. 1995; 25:358-372.

16. Platt, $\mathrm{S}$. The social transmission of parasuicide: is there a modeling effect? Crisis. 1993; 14:23-28.

17. Ovuga, E.B.L., Buga, J.W., and Guwatudde, D. Risk factors towards self-destructive behaviour among fresh students at Makerere University. East Afr. Med. J. 1995; 729:722-727.

18. Gutierrez, P., King, C.A. and Ghaziuddin, N. Adolescent attitudes about death in relation to suicidality. Suicide and Life-Threatening Behaviour. 1996; 26:8-18.

19. Neeleman, J., Wessely, S., and Lewis, G. Suicide acceptability in African and White Americans: the role of religion. The Journal of Nervous and Mental Disease. 1998; 186:12-16.

20. Gijana, E.W.M., Louw, J. and Manganyi, N.C. Thoughts about death and dying in an African sample. OMEGA. 1990; 20:245-258.

21. Wassenaar, D.R., Van der Veen, M.B.W., and Pillay, A.L. Women in cultural transition: suicidal behaviour in South African Indian women. Suicide and Life-Threatening Behaviour. 1998; 28a:82-93

22. Jilek-Aall, L. Suicidal behaviour among youth: a cross-cultural comparison. Transcultural Psychiatic Research Review. 1988; 25:87-105 